\title{
Acogida y proximidad como posibilidades de interrelación en teleterapia ocupacional: experiencia de práctica con niños y niñas del Jardín Infantil de la Universidad Nacional de Colombia
}

Reception and proximity as possibilities of interrelation in occupational teletherapy: experience from the practice with children in the Universidad Nacional de Colombia's kindergarten

Acolhimento e proximidade como possibilidades de inter-relação em teleterapia ocupacional: experiência de uma prática com crianças do Jardim de Infância da Universidade Nacional da Colốmbia

Andrea de Pilar Moreno López'

Andrés Camilo Restrepo Mora ${ }^{2}$

Katherine Lorena Blanco Munévar ${ }^{3}$

Sandra Milena Martínez Quinto ${ }^{4}$

Laura Violeta Mora Estrella ${ }^{5}$

Khristian Andrés Jaramillo Rodríguez ${ }^{6}$

Leyla Hasbleidy Sanabria Camacho ${ }^{7}$

Yaneth Carolina Ríos Gutiérrez ${ }^{8}$

${ }^{1}$ Estudiante de Terapia Ocupacional, Universidad Nacional de Colombia. Bogotá, Colombia. anpmorenolo@unal.edu.co iD https://orcid.org/0000-0002-0767-4345

${ }^{2}$ Estudiante de Terapia Ocupacional, Universidad Nacional de Colombia. Bogotá, Colombia. ancrestrepomo@unal.edu.co (iD) https://orcid.org/0000-0003-3526-1916

${ }^{3}$ Estudiante de Terapia Ocupacional, Universidad Nacional de Colombia. Bogotá, Colombia. klblancom@unal.edu.co@iD https://orcid.org/0000-0001-9747-9326

${ }^{4}$ Estudiante de Terapia Ocupacional, Universidad Nacional de Colombia. Bogotá, Colombia. smartinezq@unal.edu.co iD https://orcid.org/0000-0002-9981-8333

${ }^{5}$ Estudiante de Terapia Ocupacional, Universidad Nacional de Colombia. Bogotá, Colombia. Imorae@unal.edu.co iD https://orcid.org/0000-0002-7021-7964

${ }^{6}$ Estudiante de Terapia Ocupacional, Universidad Nacional de Colombia. Bogotá, Colombia. kjaramillo@unal.edu.co (iD) https://orcid.org/0000-0001-9621-6006

\footnotetext{
${ }^{7}$ Terapeuta ocupacional. Magíster en Musicoterapia. Máster en Psicopedagogía Clínica y Atención a la Diversidad. Docente, Universidad Nacional de Colombia. Bogotá, Colombia. Ihsanabriac@unal.edu.co

(iD) https://orcid.org/0000-0001-5509-7506
}

${ }^{8}$ Terapeuta ocupacional. Especialista en Gerencia Educativa. Magíster en Educación. Jardín Infantil, Universidad Nacional de Colombia. Bogotá, Colombia. ycriosg@unal.edu.co iD https://orcid.org/0000$\underline{0003-4518-0095}$ 


\title{
RESUMEN
}

Este artículo propone una reflexión a partir de las experiencias de un grupo de estudiantes de Terapia Ocupacional durante su práctica en el área de educación, en diálogo con las apuestas de la perspectiva ética de Lévinas y la construcción de horizontes de sentido en el campo educativo, que interpelan comprensiones de la educación como instrumento para el mero aprendizaje de habilidades y conceptos que se instalan en los sujetos. La práctica se realizó con dos grupos de niños y niñas del Jardín Infantil de la Universidad Nacional de Colombia durante el primer semestre del 2021, en la modalidad de teleterapia ocupacional. Se enfatiza en el proceso de constitución de espacios de aprendizaje y reflexión sobre el actuar profesional en la escuela, en tiempos de distanciamiento social por la pandemia de COVID-19, y de otras formas para construir relaciones basadas en la proximidad y la acogida en teleterapia.

\section{PALABRAS CLAVE}

Terapia Ocupacional, infancia, educación, aprendizaje en línea

\begin{abstract}
This article proposes a reflection based on the experiences of a group of Occupational Therapy students in their education area practice, in dialogue with the ethical perspective of Lévinas and the construction of horizons of meaning in the educational field, who question the understanding of education as a tool for the sole learning of skills and concepts installed in individuals. The practice was carried out with two groups of children in the Universidad Nacional de Colombia's kindergarten during the first semester of 2021 in the occupational teletherapy modality. Emphasis is placed on the process of creating spaces for learning and reflection on professional conducting at school, in times of social distancing due to the COVID-19 pandemic, and in other ways to build relationships based on proximity and embrace in teletherapy.
\end{abstract}

\section{KEY WORDS}

Occupational Therapy, childhood, education, online learning

\section{RESUMO}

Este artigo propõe uma reflexão a partir das experiências de um grupo de estudantes de Terapia Ocupacional, durante sua prática na área da educação, em diálogo com a perspectiva ética de Lévinas e a construção de horizontes de sentido no campo educativo, que questionam as compreensões da educação como instrumento para a mera aprendizagem de habilidades e conceitos que se instalam nos sujeitos. A prática foi realizada com dois grupos de crianças do Jardim de Infância da Universidade Nacional da Colômbia, duran- 
te o primeiro semestre de 2021, na modalidade de teleterapia ocupacional. A ênfase é colocada no processo de constituição de espaços de aprendizagem e de reflexão sobre a atuação profissional na escola, em tempos de distanciamento social devido à pandemia de COVID-19, e de outras formas para construir relações baseadas na proximidade e no acolhimento em teleterapia.

PALAVRAS-CHAVE

Terapia Ocupacional, infância, educação, aprendizagem on-line

\begin{abstract}
Aquello que ponemos en juego en el educar, es decir, lo que ofrecemos, lo que donamos, lo que intentamos poner en común, debería dirigirse a cualquiera, a esos otros que nunca pierden su carácter de desconocidos y a quienes quisiéramos recibir tanto en su generalidad como en su singularidad.
\end{abstract}

Skliar (2010, p. 25)

\section{Ancla teórica para la reflexión sobre la experiencia}

De acuerdo con Giménez (2011), la perspectiva filosófica de Emmanuel Lévinas se condensa en un humanismo basado en la comprensión y el reconocimiento del otro, a partir del cual constituimos nuestra subjetividad. Ese otro nos Ilega con la posibilidad del encuentro y el relacionamiento $y$, desde ese lugar, dibuja rutas alternas al simple interés de conocerlo y abordarlo como objeto en el cual ponemos nuestros saberes e intenciones. Una posibilidad alterna estaría dada porque, en el encuentro, nos dejemos afectar por ese otro y por su existencia, de lo que deviene un genuino interés por él: el otro me importa.
Ahora bien, aunque Lévinas no desarrolló su perspectiva ética en relación con la educación, sus aportes han dado lugar a reflexiones profundas en el campo educativo. Muchas de estas reflexiones interpelan formas verticales de relacionamiento pedagógico que buscan asimilar al otro y dialogan con alternativas que vivencien la educación como espacio de encuentro entre seres que se reconocen desde sus historias, sus subjetividades e infinitud.

En esta misma línea, Palta (2015) plantea que la educación debería concebirse como un permanente encuentro de humanidad y, por tanto, un asunto de re- 
laciones donde el aprendizaje solo tiene lugar tras el establecimiento de vínculos afectivos. Así pues, no debería reducirse a una actividad mecánica de entrega de contenidos desde relaciones unidireccionales, verticales y distantes.

Para Jaramillo et al. (2018), la educación tiene lugar como un escenario de relaciones y compromisos para con los otros y otras, un espacio para crear y tejer formas distintas de relación; por tanto, implica instantes de encuentro y proximidad. Para estos autores, la educación emerge como respuesta a la aparición y exposición de un otro, a manera de encuentro intersubjetivo en el que su vulnerabilidad se nos presenta y crea la posibilidad de hacernos cargo de él.

La forma en la que el otro se nos presenta, refiere López (2001), en Lévinas se plantea desde la metáfora del rostro, a través del cual el otro se nos muestra abiertamente, sin ocultarse $y$, aunque no lo conozco ni sé nada de él, ese encuentro cara a cara con su rostro es suficiente para reconocerlo como distinto de todo lo demás, no como objeto, sino como sujeto particular.

En este mismo sentido, la vulnerabilidad del otro también se nos presenta a través de su rostro -que refleja su singularidad-, con un nombre propio, aunque bien podría volverse un número más en la larga lista de estudiantes, pacientes, usuarios. No obstante, podemos optar por reconocerlo desde caminos distintos de relacionamiento que co-construyen el cómo, cuándo y de qué formas ofrecerse y recibirse, basado en las propias historias, experiencias y formas de ser $y$ estar en el mundo. Jaramillo et al. (2018) dirán que "la escuela es un escenario educativo donde ocurre la enseñanza en clave de proximidad y acogida" (p. 5-6), como posibilidades de relacionamiento con el otro.

La acogida, en el sentido de recibir al otro, es visible en relacionamientos en los que se da lugar al decir y el dejar decir, en el deseo de estar ahí para el otro, de recibirlo en su singularidad, al recibir del otro en términos de ser enseñado por él. La familia y posteriormente la escuela serán los primeros espacios de acogida en los que el encuentro con unas y unos prójimos propende por el cuidado y la socialización.

De esta manera, el encuentro con prójimos cercanos se presenta como las distintas formas de proximidad con el otro. Refieren Jaramillo et al. (2018) que, en Lévinas, la proximidad es recibimiento y acogida del otro en su total alteridad, en la posibilidad de dejarse permear por sus historias, sus silencios y decires, en pocas palabras, por su humanidad. En este sentido, en el espacio educativo, la proximidad tendrá relación con un mirar-se en las formas e intereses de aproximación al otro -que trascienden la transmisión mecánica de contenidos- y se preocupa además por el para qué de las enseñanzas y de las formas en que tienen lugar. Jaramillo y Murcia (2014) lo plantean en términos de una pedagogía del encuentro que implica desprenderse de discursos tradicionales que reconocen al otro como objeto.

Para Jaramillo et al. (2018), tanto la acogida como la proximidad ponen relevancia en el encuentro y en la subjetividad que se construye cuando me hago consciente de la presencia del otro. Sin conocerlo, me importa. $\mathrm{Y}$ aunque ya nombrado como usuario, paciente, estudiante, lo desbautizo para tomar dis- 
tancia de pretender normalizar $\mathrm{u}$ homogeneizar, dando paso a conocer sus historias encarnadas, su nombre, su vOz, sus temores y sentires.

Hasta aquí, hemos referido brevemente la acogida y la proximidad como conceptos clave desde la perspectiva ética de Lévinas, como puerta de entrada para compartir la experiencia de una práctica profesional integral en educación, realizada en la modalidad de teleterapia Ocupacional durante el primer semestre de 2021 en la Universidad Nacional de Colombia. A partir de allí, nos planteamos la pregunta: ¿qué tipo de vínculos y acciones se co-construyeron en la experiencia de práctica profesional con un grupo de niños y niñas de 2 y 3 años de edad en el Jardín infantil de la Universidad Nacional de Colombia?

\section{Presentación de la experiencia: levantar las anclas, navegar mar adentro en teleterapia ocupacional}

A principios del año 2020, la pandemia de COVID-19 trajo consigo una serie de desafíos personales y sociales que incluyeron el ámbito académico y movilizaron reflexiones sobre el papel social de la educación y la necesidad de recrear las estrategias educativas. En educación superior, y en particular en el programa de Terapia Ocupacional de la Universidad Nacional de Colombia, uno de los mayores retos se dio en los espacios de práctica, dadas las estructuras pedagógicas y relacionales construidas históricamente con las y los usuarios, la inexperiencia en el uso de la teleterapia y los desafíos de la brecha digital en estudiantes de una universidad pública.
Para mediados del 2021 se determinó el reinicio de las prácticas profesionales del programa de Terapia Ocupacional. En el área educativa se desarrolló una de ellas, en el Jardín Infantil de la Universidad Nacional de Colombia. En este espacio se implementaron estrategias de acompañamiento y encuentro mediadas por la virtualidad, con los hijos e hijas de docentes, trabajadores, trabajadoras y estudiantes de la Universidad que asisten a la institución.

¿Cómo podríamos construir una práctica completamente virtual con niños y niñas de 2 y 3 años? ¿Cómo establecer vínculos con niñas y niños tan pequeños, manteniendo el sello particular de las acciones profesionales a través de actividades con propósito para favorecer su bienestar ocupacional en medio de una pandemia? Estas fueron algunas de las preguntas a partir de las cuales el grupo de estudiantes, la docente de práctica y la terapeuta ocupacional del jardín empezamos a pensar en posibilidades que pasaron por la revisión de literatura en torno al ejercicio profesional en teleterapia (Federación Mundial de Terapeutas Ocupacionales [WFOT, por sus iniciales en inglés], 2014; Olivera, 2020; Peñaloza et al., 2020) y culminaron con el diseño e implementación de un programa de promoción dirigido a dos grupos de niños y niñas del grado prejardín.

De acuerdo con los lineamientos del Colegio Colombiano de Terapia Ocupacional (Peñaloza et al., 2020), las acciones para la telesalud -nombradas en este artículo como teleterapia- deben garantizar la atención plena para niños, niñas y adolescentes durante las restricciones de presencialidad y en las dinámicas de la pandemia por COVID-19. En consecuencia, frente a la imposibilidad de contacto 
directo con la población, el uso de tecnologías de la información, a partir de sus plurales mecanismos, se constituye en una estrategia central en los procesos de teleterapia a partir de dos modalidades:

Modalidad interactiva o interacciones sincrónicas: hace referencia a las actividades ejecutadas en tiempo real entre terapeuta y usuario/usuaria a través de estrategias como plataformas virtuales, juegos, entre otras.

Interacciones asincrónicas: en esta modalidad la o el terapeuta a cargo acompaña a la persona a través de videos, fotografías, mensajes, infografías, actividades para desarrollar en casa, entre otros recursos.

Con estas claridades conceptuales, definimos una serie de acciones a desarrollar en dos fases:

En la primera fase se recopiló información para la lectura de potencialidades y necesidades de los grupos de niños y niñas. Este proceso se realizó a través de la revisión de los resultados de la Escala Abreviada del Desarrollo 3 (Pontificia Universidad Javeriana, Facultad de Medicina, s.f.), aplicada por el grupo interdisciplinario del jardín, y el uso de la Prueba de Actividades Básicas Cotidianas Infantiles (Urrea, 1980) ${ }^{9}$. Esta última recoge información sobre el desempeño individual, a través de entrevista con cuidadoras o cuidadores; observación directa en actividades de alimentación, higiene personal, vestido y sueño, y el levantamiento de información comple- mentaria a través de preguntas abiertas a padres, madres o cuidadoras en la herramienta Google Forms.

Contábamos con dos grupos de niños y niñas entre 2 y 3 años que no habían tenido la experiencia de presencialidad en el jardín ni de actividades escolares, pues para la mayoría era su primer año de escolarización. Adicionalmente, sus familias estaban expuestas a una serie de cambios en la vida familiar y laboral, sumados a los retos del cuidado permanente de sus hijos e hijas.

Los resultados de la primera fase fueron llamativos en relación con la desestructuración de rutinas y hábitos de sueño de los niños y las niñas; las dificultades para la participación e independencia en actividades de alimentación, vestido e higiene, acordes con la edad, y la necesidad de fortalecer las habilidades motoras, adaptativas y personal-sociales.

A partir de estos hallazgos, se determinaron como objetivos del programa de promoción los siguientes: favorecer el bienestar ocupacional y la maduración de habilidades de los niños y niñas mediante la participación en actividades básicas cotidianas significativas; diseñar e implementar herramientas digitales para la difusión de información dirigida a familias o cuidadores/cuidadoras como complemento a las actividades directas con los niños y niñas, y desarrollar actividades de acompañamiento individual en los casos que así lo requirieran.

\footnotetext{
${ }^{9}$ Prueba semiestructurada elaborada por la docente María Victoria Urrea, como parte de proyectos académicos interdisciplinarios realizados en la década de 1980 entre la Universidad Nacional de Colombia y el Instituto Colombiano de Bienestar Familiar (ICBF). Actualmente, se emplea en los espacios de práctica profesional integral en la Universidad Nacional de Colombia. Se requieren y recomiendan estudios para su validación y desarrollo.
} 
La segunda fase consistió en la construcción de la estrategia para el desarrollo del programa y su implementación a partir de los insumos recopilados en la fase anterior. En este contexto, teníamos el desafío de diseñar espacios, acciones e interacciones que incentivaran la participación de niños y niñas de 2 y 3 años, con procesos madurativos y escolares particulares.

Motivados por este interés nació Teo el león y sus amigos, una estrategia didáctica para el desarrollo del programa de Terapia Ocupacional. Durante una sesión semanal, ocho personajes de la selva, personificados por las y los terapeutas en formación, experimentaban situaciones de la vida cotidiana e invitaban a las niñas y los niños a vincularse en diversas actividades para ayudarles y enseñarles desde sus propias experiencias.

Cada sesión sincrónica fue diseñada reconociendo la necesidad de acompañar los procesos de desarrollo infantil y participación ocupacional desde el afecto, la empatía y la creación de espacios seguros que promovieran la exploración de gustos e intereses y la libre expresión de los niños y las niñas. La estructura de cada sesión incluía actividades psicomotoras y de autorregulación para el inicio; una actividad central relacionada con actividades básicas cotidianas infantiles, a través de una aventura de los animales en la que los niños y las niñas los apoyaban para resolver distintas situaciones, $y$ una actividad de cierre.

Como complemento a los encuentros sincrónicos semanales se creó un sitio web con actividades asincrónicas relacionadas con las temáticas tratadas. Estas actividades estuvieron dirigidas a los niños, las niñas y sus familias, con el ánimo de fortalecer las habilidades abordadas en cada sesión y propiciar la interacción familiar a través del juego.

Durante 12 semanas se desarrolló el programa y se consolidaron relaciones basadas en la empatía y el vínculo afectivo entre niños, niñas y terapeutas en formación. La asistencia de los niños y las niñas a las sesiones, así como la vinculación de las familias en las actividades fueron variadas y fluctuantes a lo largo del proceso, lo que permitió develar particularidades en las relaciones familiares; los contextos sociales de los niños y niñas; el impacto del distanciamiento social en la participación ocupacional, y las formas diversas de interacción y vinculación con el programa. El reconocimiento de estas realidades y la legitimación de las formas específicas de expresión de cada niño o niña y sus familias fueron claves en el proceso de entender nuestro lugar en el acompañamiento como terapeutas ocupacionales en medio de una pandemia y como gestores o gestoras sociales de cambio, desde el acoger y aproximarnos a quienes nos permitieron entrar en sus espacios vitales.

Entre los logros del programa, se consolidaron procesos de acompañamiento a las familias para favorecer la participación de los niños y las niñas en actividades básicas cotidianas. Con el desarrollo del sitio web, por ejemplo, se proporcionaron opciones complementarias para promover los procesos madurativos en habilidades cognitivas, perceptuales y motoras, enfocadas en el desarrollo de actividades cotidianas de alimentación, vestido e higiene, y la organización de hábitos y rutinas de sueño de los niños y las niñas. Adicionalmente, se apoyaron aquellos casos individuales que requirieron mayor acompañamiento terapéutico. 
Es necesario reconocer también las dificultades implícitas en el uso de la virtualidad: la inestabilidad en las conexiones a internet o las restricciones en el acceso y uso de dispositivos electrónicos inteligentes, que para algunas familias no eran asequibles. Frente a estos retos se definieron estrategias complementarias como la creación de micromensajes animados para motivar la participación de los niños y las niñas; la disposición de materiales de uso asincrónico, recordatorios de las sesiones, enlaces a sitios web y otros recursos; así como el ajuste de horarios para convocar el mayor número posible de participantes.

\section{Entre diálogos de la experiencia}

Movilizar nuestro actuar profesional en el marco de una pandemia resultó en una experiencia con múltiples aristas de reflexión. Por un lado, volver la mirada sobre las formas en las que, como terapeutas ocupacionales, definimos unos objetivos terapéuticos y unos procesos de atención en las que el otro puede quedar enajenado, a menos que permitamos que su historia marque la diferencia para establecer un acercamiento ético en el que nos hacemos responsables por él y reconocemos su subjetividad, su otredad.

En este acercamiento ético, acoger y aproximarse a otro, desde sus propias resistencias y formas particulares de ser, puede implicar encontrarse con comportamientos que no logramos entender, o que debamos ajustar toda la planeación de la sesión terapéutica a partir de sus necesidades en un aquí y un ahora. Esto significa movilizarnos hacia un actuar que nos llama a recuperar otras formas de interrelación... le dejo ser para reco- nocerle, me encuentro con su más sincera expresión, así no se ajuste a lo que yo espero y deseo como terapeuta, es decir, si no cede al riesgo de volverlo otro yo, el mí mismo.

Los niños y las niñas, como otro, llegaban a nuestro encuentro desde sus contextos; con sus deseos y aspiraciones de conocer el mundo, de interactuar y dar-se; como prójimos en el sentido de próximos, cercanos, conocidos en el marco de las acciones posibles dentro de una práctica de formación profesional. Sus rostros aparecen a través de una pantalla, lo que no minimiza las posibilidades de reconocerles y demandar una genuina responsabilidad y preocupación por cada uno, cada una... Se conectaban esperando ver a los animales, jugar con ellos, sentirse escuchados, acompañados y validados. La validación de los niños y las niñas se expresaba no solo en los espacios de encuentro, donde les brindábamos toda nuestra atención, sino en todas las acciones previas de planeación, ajuste, modificación o graduación de las actividades, pensando en las posibilidades y barreras que podrían encontrar, esto es, en su vulnerabilidad.

Es claro que la planeación y la implementación de las actividades requería procesos juiciosos de razonamiento profesional y también de tensión frente a lo que se espera de los niños y las niñas en respuesta al proceso terapéutico. En este sentido, Santos (citado por Iza, 2018) plantea que el conocimiento es el lenguaje del mundo académico, y la racionalidad, una de sus principales características; pero este se ha centrado y consolidado en el pensar, antes de existir, negando lo que somos por naturaleza. Desde este conocimiento académico se nos impone ver al otro como objeto de 
interés, de estudio, de evaluación, de rehabilitación, de intervención, y se desdibuja su singularidad y trascendencia.

Así que el llamado de Lévinas pasa por la ética de las relaciones y cuestiona cualquier intención de absorber al otro y limitarlo en su infinitud, definiendo lo que espero que sea y haga desde mi lugar de poder como terapeuta. Pero también, abre la posibilidad de que el reconocimiento del otro devele lo que soy, me permita ser consciente de lo que siento, lo que deseo, lo que pienso, por lo que el otro me permite descubrirme en la medida en que, ante la presencia de su rostro, le permito simplemente ser. En palabras de Iza (2018), la otredad se vuelve mismidad.

Un segundo punto importante se relaciona con los enfoques base de la estrategia: la construcción de relaciones desde el acogimiento y la proximidad, que se traducen en el afecto, el cuidado, la escucha activa y la empatía. Estas características hacen que la praxis de las y los terapeutas ocupacionales necesariamente se centre en la persona a partir de sus fortalezas y necesidades, su historia ocupacional y su identidad. De esta manera podemos expresar que ese otro nos importa y exponemos nuestro genuino interés por una búsqueda de acciones profesionales, emocionales, terapéuticas, que impacten en su bienestar y en su participación ocupacional. Mientras nuestras prácticas no incluyan ejercicios reflexivos de reconocimiento del otro, estaremos destinados al olvido del yo, como lo refiere López (2001), y con ello al desconocimiento de su otredad y de la trascendencia en su vida.

En tercer lugar, se destaca la riqueza que para la práctica de Terapia Ocupa- cional implicó contar con dos grupos del Jardín Infantil conformados por niños y niñas de diferentes regiones del país, con particularidades culturales y sociales, lo que nos permitió tejer experiencias desde la diversidad.

En tiempos de pandemia, las dinámicas de vida cambiaron drásticamente, las restricciones y el distanciamiento físico se convirtieron en barreras para poder compartir con otros y otras en espacios educativos como el jardín infantil. Sumado a ello, las familias se enfrentaron a nuevas dinámicas en las que el espacio del hogar fue abocado a múltiples ocupaciones y roles que no se realizaban habitualmente en casa, lo que implicó tensiones. A pesar de ello, el programa desarrollado permitió que desde los encuentros virtuales se entablaran vínculos afectivos entre personas desconocidas, entre pares y con las profesoras, además de crear espacios para la exploración de gustos, intereses y potencialidades.

Esto implicó retos para estudiantes, docentes, cuidadoras y cuidadores en la adaptación a las tecnologías y a las formas de interacción que estas demandan, por ejemplo, la intermediación de una pantalla que no permite sentir físicamente al otro. Los dispositivos electrónicos y plataformas virtuales, hasta entonces poco explorados, requirieron la adquisición de nuevos conocimientos sobre su funcionamiento y uso. Para practicantes, maestras, niños, niñas y familiares, la virtualidad supuso irrumpir en su intimidad, permitir a otras personas conocer sus entornos familiares, sus historias personales, sus facilidades y restricciones en el acceso a las tecnologías. Todo ello, desde la perspectiva de Lévinas, nos pone frente al otro desde nuestras vulnerabilidades. 
En este escenario, como estudiantes de Terapia Ocupacional asumimos grandes desafíos, miedos y dudas respecto a cómo adaptarnos a una nueva realidad digital que no era posible anticipar. Aun así, con muchas expectativas e ideas, comenzamos a recorrer un camino inexplorado que nos llevó a encontrar nuevos aprendizajes frente al uso de las tecnologías de la información y la comunicación en teleterapia y nos condujo a alcanzar resultados significativos en el proceso de cada niño o niña y su familia, descubriendo y fortaleciendo habilidades para nuestra labor futura como terapeutas ocupacionales.

Finalmente, el programa se constituyó en un espacio de acogimiento, de transformación y de reconocimiento de los niños y las niñas como sujetos de saberes. Si bien los conocimientos sobre desarrollo infantil son importantes para el quehacer profesional, no deberían ser la única fuente de saber, deben ser interpelados por la presencia de los niños y las niñas como evidencia de otras formas de aprender y comprender el desarrollo infantil, un desarrollo encarnado, situado y constituido desde sus particularidades.

\section{Reflexiones finales}

Es necesario pensar, desde los procesos de formación, en tensionar las formas de relacionamiento centradas en cosificar al otro, que se sustentan en relaciones distantes y mecánicas, para dar paso a otras que favorezcan la acogida y la proximidad.

Entender al otro no solo como paciente o usuario de forma aislada, sino como sujeto que se nos revela desde sus historias, situaciones y vivencias, y que está permeado por su entorno y por condiciones económicas, políticas, sociales. Un otro con múltiples formas de ser en el mundo y de experimentar la ocupación.

Es menester comprender que, como parte del complejo y dinámico proceso de aprendizaje mutuo que tuvo lugar durante esta experiencia, se revelaron dificultades en la proximidad en los espacios de interacción terapéutica. Esto no solo se debe a la actual contingencia por COVID-19, sino a las formas tradicionales de relación con el otro que, limitadas a lo físico o presencial, desconocen otros modos de interacción a los cuales precipitadamente hemos sido convocados como terapeutas ocupacionales, maestros y maestras, estudiantes, como individuos, y que requieren nuevas maneras de entender lo próximo y lo cercano.

Esta experiencia permitió la exploración personal de las y los participantes: terapeutas, niños y niñas, familias y educadoras que hicieron parte del proceso, a través de procesos de introspección y aprendizaje sobre habilidades, intereses, relaciones interpersonales y aspectos emocionales asociados con las nuevas formas de ocuparnos, con la realidad actual y la terapia llevada a cabo desde la virtualidad, que replantea la relación con el otro.

Asimismo, para las y los terapeutas ocupacionales que participamos (docentes y estudiantes), el espacio de la práctica se construyó desde la empatía, la solidaridad, el compañerismo y el trabajo en equipo, situando las capacidades y habilidades de cada uno y cada una como potenciadores en los distintos encuentros con los niños y niñas. Por lo tanto, explorar mediante la corporalidad, la gestuali- 
dad, el tono de voz, la improvisación y, en sí misma, la oportunidad de entablar una relación terapéutica a través de una plataforma virtual, fortaleció los procesos de aprendizaje como terapeutas ocupacionales en el área de educación.

Transitar por primera vez una práctica virtual en la carrera de Terapia Ocupacional de la Universidad Nacional se convirtió en un reto constante. La recursividad y la creatividad fueron la base de cada uno de los espacios, permitiéndonos observar diferentes aspectos en la cotidianidad de los niños y las niñas, y crear estrategias para favorecer su participación y bienestar ocupacional, vinculando a las familias y maestras.

Los resultados de la práctica nos llaman a reconocer la importancia de educar desde los afectos y la empatía. Esto se vio materializado en los procesos de enseñanza, dado que todos los encuentros se tejieron con base en la comprensión y la escucha, convirtiéndose en lugares seguros y humanos que le apostaron, no solo a agenciar las relaciones entre profesoras y estudiantes, sino a construir la Terapia Ocupacional desde los afectos.

Finalmente, queremos resaltar la necesidad de replicar prácticas que conlleven a fortalecer la relación terapéutica desde la acogida y la proximidad, aun más con población infantil, teniendo en cuenta que la niñez es la base de los vínculos y las emociones de las personas. La experiencia que compartimos en este artículo invita a las y los terapeutas ocupacionales a repensar la cotidianidad y, con ello, las maneras en que se fortalece la empatía y la solidaridad con las personas que acompañamos en nuestros quehaceres.

\section{Referencias}

Federación Mundial de Terapeutas Ocupacionales [WFOT] (2014). Declaración de posicionamiento. Telesalud. WFOT. https:// wfot.org/resources/occupational-therapy-and-telehealth

Giménez, A. (2011). Emmanuel Lévinas: humanismo del rostro. Escritos, 19(.43), 337-349.

Iza, V. A. (2018). El rostro y la otredad de Emmanuel Lévinas como elementos de alteridad y su implicación en el personalismo cristiano. En V.A.Iza (ed.), Persona, educación y filosofía: reflexiones desde la educación universitaria (pp. 67-84). Editorial Abya-Yala. https://doi. org/10.7476/9789978104934.0005

Jaramillo, D. y Murcia, N. (2014). Hacia una pedagogía del encuentro: apuesta por la relación cuerpo-alteridad en educación. Revista de Investigaciones UCM, 14(24), 142-149. http://dx.doi.org/10.22383/ri.v14i2.28

Jaramillo, D., Jaramillo, L. y Murcia, N. (2018). Acogida y proximidad: algunos aportes de Emmanuel Lévinas a la educación. Revista actualidades investigativas en educación, 18 (1), 1-16. http://dx.doi.org/10.15517/ aie.v18i1.31771

López, M. (2001). El otro en la filosofía de Lévinas. Investigaciones fenomenológicas, (3), 265-282. https://doi.org/10.5944/ rif.3.2001.5430

Olivera, R. (2020). The Telehealth OT. A guide to teach occupational therapists about telehealth. Publicación independiente. 
Palta, W. (2015, 11 de mayo). La proximidad como compromiso ético del maestro. WiIlian Fredy Palta Velasco [blog]. https:// wpalta.blogia.com/2015/051102-la-proximidad-como-compromiso-tico-del-maestro.php

Peñaloza, E., Chavarro, A., Ardila, C., Rios, J., Watemberg, J., González, M., Rodríguez, E. y Niño, R. (2020). Lineamientos generales de terapia ocupacional para la atención de niñas, niños y adolescentes durante la pandemia y pospandemia por COVID-19 en Colombia. Colegio Colombiano de Terapia Ocupacional. https://doi. org/10.25214/ccto. 2

Pontificia Universidad Javeriana, Facultad de Medicina (s.f.). Escala abreviada del desarrollo 3. Ministerio de Salud y Protección Social. https://www.minsalud.gov.co/sites/ rid/Lists/BibliotecaDigital/RIDE/VS/PP/ ENT/Escala-abreviada-de-desarrollo-3.pdf

Skliar, C. (2010). Pedagogía de las diferencias. Noveduc.

Urrea, M. V. (1980). Prueba de Actividades Básicas Cotidianas Infantiles [documento de trabajo]. Departamento de la Ocupación Humana, Universidad Nacional de Colombia. 